Kryokonservierung von Oozyten. Schon 1986 wurde von der ersten erfolgreichen Schwangerschaft nach Kryokonservierung der Eizellen berichtet. Das Problem dieses Verfahrens liegt in der äußerst anfälligen Struktur des Zytoskeletts von Metaphase-II-Oozyten, welches beim Einfrier- und Auftauvorgang empfindlich geschädigt werden kann. Daneben spielt die Schädigung des Spindelapparats eine entscheidende Rolle für das Zellüberleben. Bei der oben beschriebenen Entwicklung der Vitrifikation konnten Zellüberlebensraten von über $80 \%$ beobachtet werden $[3,12]$, im Vergleich $\mathrm{zu}$ Raten von $50-65 \%$ bei Einsatz des „slow rate freezing".

Obwohl die Kryokonservierung von menschlichen Eizellen nach wie vor als experimentelle Methode angesehen wird, geben aktuelle Studienergebnisse Hoffnung auf einen klinischen Einsatz in naher Zukunft. Diese Methode scheint gerade für junge Patientinnen ohne festen Partner zum Zeitpunkt der Diagnosestellung, für die eine donogene Samenspende nicht in Frage kommt, von Vorteil.

Eizellgewinnung. Jegliche Kryokonservierung menschlicher Eizellen oder Embryonen bedarf der vorherigen Eizellgewinnung.

Stimulation mit Gonadotropinen: Ovarielle Stimulationsprotokolle führen durch Gabe von Gonadotropinen zu supraphysiologisch hohen Serum-FSH- und Östrogenwerten. Der Einfluss der Östrogene gerade auf hormonsensitive Tumoren besonders der Brust und des Endometriums wurde in der Vergangenheit durch mehrere Arbeiten [26, 29] hinlänglich beschrieben. Ovarielle Stimulationsprotokolle können somit lediglich bei $\mathrm{Pa}$ tientinnen mit negativem Hormonrezeptorstatus angewandt werden. Zudem ist diese Möglichkeit nur gegeben, wenn ausreichend Zeit für die Stimulation vorhanden ist und eine onkologische Therapie nicht direkt initiiert werden muss.

Bei Vorliegen hormonsensitiver Tumoren sind alternative Strategien unumgänglich. Hierzu zählen Stimula- tionsversuche mit Aromatasehemmern oder Antiöstrogenen (allein oder in Kombination mit rekombinantem FSH) zusammen mit GnRH-Agonisten, die eine Reifung der Eizellen bedingen sollen. Erste Studienergebnisse hierzu waren positiv [18]. Weitere experimentelle Therapieansätze basieren auf der Verwendung von kurzen ovariellen Stimulationsprotokollen, der IVM oder ovariellen Kortexgewinnung mit anschließender Kryokonservierung (s. unten).

Kurze und ultrakurze Stimulationsprotokolle: Auch die notfallmäßige ovarielle Stimulation im Rahmen kurzer und ultrakurzer Stimulationsprotokolle setzt die Hormonrezeptornegativität des Tumors voraus. Dabei kann in nur kurzer Zeit ein Fertilitätserhalt gewährleistet werden, wobei eine dringende Chemotherapie oder Operation zeitlich nicht verschoben werden müssen. Mittlerweile existieren zyklusunabhängige Stimulationsprotokolle auf der Basis von GnRH-Antagonisten, die direkt bei der Patientin zur Anwendung kommen können.

IVM: Hierunter versteht man die Gewinnung unreifer Eizellen, welche anschließend unter Laborbedingungen die erste Reifeteilung beenden. Somit lassen sich sämtliche Stimulationsprotokolle umgehen. Diese Methode kann jederzeit angewandt werden und führt demzufolge zu keinem Zeitverlust in der Initiierung einer onkologischen Therapie. Ihre Durchführung ist nach wie vor Studien vorbehalten.

Erfolgsraten zeigen immer noch deutliche Unterschiede. So wurden Fehlbildungsraten nach IVM von bis zu $37 \%$ beschrieben, wobei dieser hohe Prozentsatz wahrscheinlich eher auf das untersuchte Kollektiv von PCOS-Patientinnen als auf die Methode der IVM selbst zurückzuführen ist. Auch Lebendgeburten nach Vitrifikation von in vitro maturierten und anschließend befruchteten Eizellen wurden beschrieben [2]. Im Vergleich zwischen in vivo und in vitro gereiften Eizellen zeigen die natürlich gereiften Oozyten signifikant bessere Erfolgsraten für das Zellüberleben, die Rate der

\title{
Kinderwunsch trotz Krebserkrankung
}

\section{Die Stiftung Gesundheit hat den Ratgeber „Krebs und Kinder- wunsch: 100 Fragen - 100 Antwor- ten" geprüft und zertifiziert.}

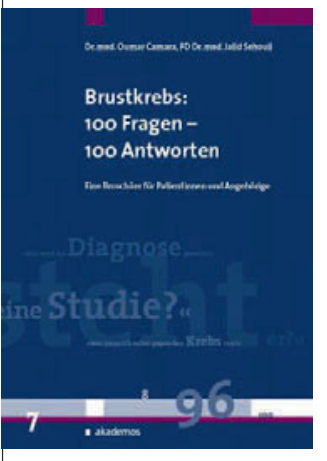

Mit freundlicher Genehmigung der akademos Wissenschaftsverlag $\mathrm{GmbH}$
Diagnose Krebs: Für jeden Patienten ist es zumeist ein Schock. Selbst nach erfolgreicher Heilung bleibt bei Frauen im gebärfähigen Alter oft eine unerwünschte Nachwirkung der Krankheit:

die Einschränkung oder gar der Verlust der Fruchtbarkeit. Auch Männer sind betroffen: Bereits bei der Diagnosestellung Hodenkrebs liegt eine eingeschränkte Fruchtbarkeit vor und die Chemotherapie vermindert diese zusätzlich. Der Patientenratgeber zeigt Paaren Wege auf, wie ein Kinderwunsch trotz vorangehender Krebserkrankung erfüllt werden kann.

In dem Werk werden 100 Fragen thematisiert und beantwortet, die sich mit dem Thema Krebs und Kinderwunsch befassen. Dabei wird im ersten Teil besonders auf Fragen zur Krankheit und die Auswirkungen von Behandlungsmethoden auf die Fruchtbarkeit eingegangen. Im Anschluss zeigen die Autoren, welche Möglichkeiten bestehen, trotz eingeschränkter Fruchtbarkeit den Kinderwunsch doch noch zu erfüllen: von künstlicher Befruchtung bis hin zur Adoption.

„Krebs und Kinderwunsch: 100 Fragen - 100 Antworten" (2009)

Autoren: K.J. Bühling, A. Jantke,

J. Sehouli

Akademos Wissenschaftsverlag, 66 Seiten, ISBN 978-3-86748-008-6

Quelle: Stiftung Gesundheit, www.stiftung-gesundheit.de 